\title{
Specifics of biomedical parameters estimation of human organism respiratory subsystem
}

\author{
Kosheva Larysa' (iD, Ivanets Olha² \\ ${ }^{1,2}$ National Aviation University, Kyiv, Ukraine \\ E-mail:olchik2104@ukr.net
}

\begin{abstract}
Offered approach to the functional state of organism estimation as a holistic system with the complex of interrelated, interactive, mutually affecting functional subsystems of homeostatic type, which may be described as statistically stable biomedical parameters complex. Offered integral index helps to estimate the respiratory subsystem condition using basic organism indices. Information model of the biomedical parameters transformation was developed. Input quantities uncertainty analysis of the mentioned subsystem information model of the organism gave an opportunity to increase probability of making correct decisions concerning the biological objects condition. Methodic of the respiratory subsystem condition estimation was developed and possible conditions range was determined.
\end{abstract}

Keywords: respiratory subsystem, integral index, functional condition, mathematical model, measurement results uncertainty, indices variability, decision making.

\section{Introduction}

One of the main goals in the health care sector is the organism functional condition estimation technology development. The body functional condition analysis is necessary for correct conclusion in the process of diseases diagnostics, current functional condition analysis, therapy methods choice, rehabilitation process support, requirements of personal and social safety securing in the process of professional activities in extreme conditions, professional competence estimation and many other tasks decision.

\section{Sources overview}

Human body consists of many systems having close connection to each other and due to the homeostasis are in balanced conditions. Each subsystem analysis gives an opportunity to estimate the whole body functioning and prognosticate its condition in future [1]. Variability is a specific and distinctive characteristic peculiar to the biomedical measurements of almost all organism parameters. Characteristic variation appears when its value changes from one individual to another one or temporally for one individual. It is almost impossible to provide the same parameters of the same organism second control measurements, as it is used to do in technique that leads to the great difficulties in the process of random values evaluation and as a result, the diagnostic errors appear [2]. In this paper as the organism subsystem is described the respiratory subsystem, which is a very important part of the homeostasis. In the introduced paper analysis of the literary and patent researches is provided, which describes the way of the respiratory apparatus condition evaluation using different indices. Thus, among foreign scientists papers there should be marked out the following researches of Harrison-Benedict and Wattchal-Tiffano, who developed tables for calculation of the ventilation equivalent taking into account weight, height and analyzing metabolic rate using energy loss indices for tracheobronchial patency estimation. Among national researchers, there should be mentioned development of the ways to estimate the 
respiratory subsystem functional condition level [3]. Based on the sources overview, the integrated method to biomedical parameters evaluation was introduced.

\section{Measurement model for biomedical parameters of human organism respiratory subsystem evaluation}

Based on the systematic approach human organism is considered as the complicated interrelated system including main subsystems of the organism such as cardiovascular, respiratory, digestive, locomotive, endocrine, nervous and urogenital [3]. Research of the functional specifics and each subsystem role determination and their mutual effects will afford to increase accuracy of the functional condition estimation of the whole body. Ways of the variability parameters analysis of the whole organism as the variable biological object taking into account technical specifics of the diagnostic instruments and diagnostic methods is a rather difficult task. Consequently, in this paper is considered one of the body subsystems for example, the respiratory one.

The aim of this publication is to prove the possibility of biomedical parameters of human organism respiratory system evaluation according to the developed model of this evaluation.

The following equation is offered as the integral index of the respiratory subsystem functional condition estimation [3]:

$$
Y=100 \cdot \frac{A \cdot B-C \cdot D}{A}
$$

where

A - maximum ventilation of lungs (MVL);

B - hypoxia index;

C - Skibinski index;

D - Harrison Ventilation Index.

Model for respiratory subsystem functional condition evaluation based on the integral index is presented on the Fig. 1.

According to the model equation (1) ventilation index is calculated by the formula:

$$
D=\frac{x_{2}}{x_{0}}
$$

where

$x_{2}$ - minute volume of breath (MVB);

$x_{0}$ - actual living capacity of lungs (ALCL).

Ventilation index value may be calculated as the criterion of the potential capabilities for the external respiratory system of the particular individual.

Skibinski index is calculated in the following way:

$$
C=x_{0} \frac{x_{1}}{x_{4}}
$$

where

$x_{1}$ - time of breath exhalation $\left(\mathrm{T}_{\text {exhal }}\right)$
$x_{4}$ - heart rate (HR).

External factors (stress, ecology, etc.)

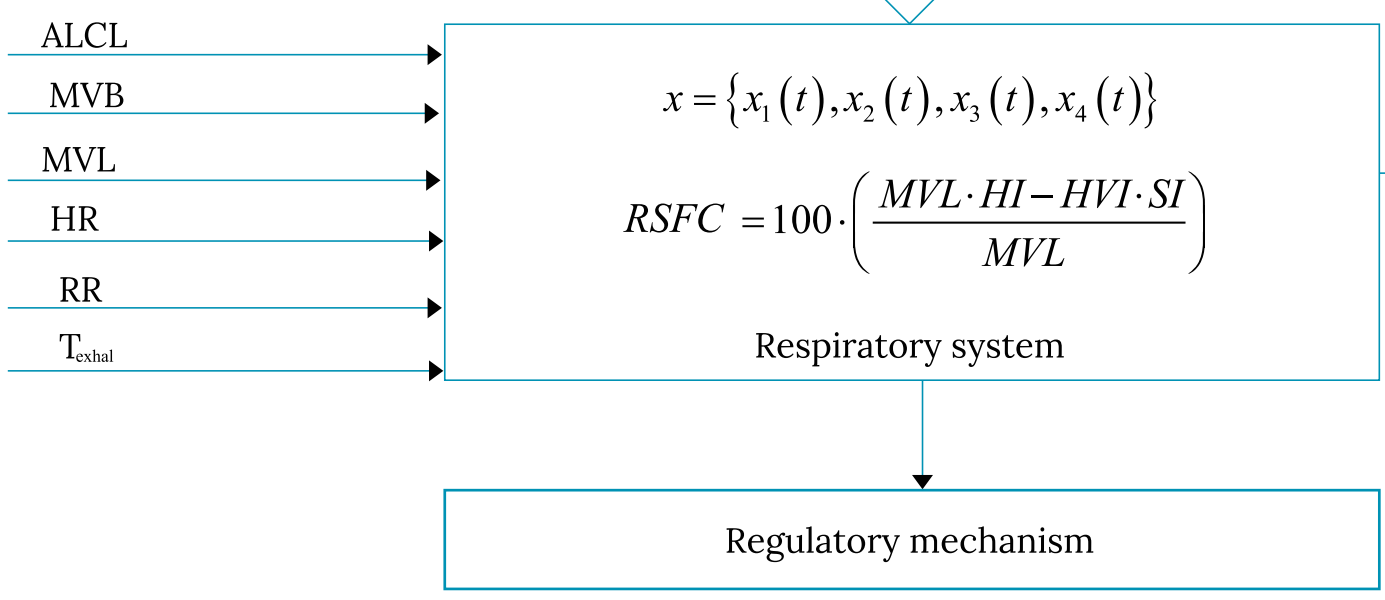

1-st integral index for respiratory system condition estimation

Fig. 1. Model of the respiratory subsystem functional condition evaluation 
Skibinski index characterizes not only potential capabilities of the external respiratory system, its resistance to hypoxia and, to a great extent, coordination of the respiratory subsystem functioning with cardiovascular organism subsystem. Hypoxia index is traditionally calculated by the formula:

$$
B=\frac{x_{1}}{x_{4}} .
$$

This index characterizes the resistance degree of the body to the oxygen deficiency.

Respiratory system is closely connected with the cardiovascular system. Thus, volume of breath rate is connected directly to respiratory rate (RR) and heart rate. Heart rate and respiratory rate were evaluated for that purpose.

Thus, mathematical model (1) will be the following:

$$
y=\frac{x_{1} \cdot\left(x_{3}-x_{2}\right)}{x_{4} \cdot x_{3}},
$$

where $x_{3}$ - maximum ventilation of lungs (MVL) [3].

Mathematical equation (5) is basic for development of the expanded information model for decision making concerning the respiratory system condition, presented on the Fig. 2.

Block of converted measurement values provides conversion of the indices $x_{1}{ }^{*}, \ldots, x_{k}{ }^{*}$ of the controlled values using Spirograph SP100 HEACO and tonometer OMRON MIT3.

Block of converted measurement values provides conversion of $x_{1}{ }^{*}, \ldots, x_{k}{ }^{*}$ to estimation $Y^{*}$ parameter value of $Y$ using mathematical conversion model:

$$
M[Y]=F\left(M\left[X_{1}\right], \ldots, M\left[X_{k}\right]\right) .
$$

Respiratory system condition evaluation is provided in the phase of diagnostic object examination according to sampling with the volume $n$ for each of the fixed values $Y_{j}, j=\overline{1, m}$ of $Y$ parameters level.

Block of decision making chooses $\left\{a_{l}, b_{c}\right\}^{m}$ of one $y_{j}\left\{a_{l}, b_{c}\right\}_{l}^{m}$ from quantity $\left\{y_{l}\right\}_{l}^{m}$ decisions concerning the respiratory system condition index $Y$ (q.v. Fig.2) after comparing $Y^{*}$ to generally accepted rate for each examined group $\left(a_{l}, b_{l}\right), l=\overline{1, m}$ according to the decision making rule $[4,5]$ :

$$
\forall Y^{*}\left[Y^{*} \in\left(a_{j}, b_{j}\right) \rightarrow Y^{*} \in Y_{j}\right] .
$$

Quantity information evaluation about $Y$ parameter is provided on condition that $\Delta$ width of confidence intervals $\left(a_{j}, b_{j}\right), j=\overline{(1, k)}$ is equal and general quantity is equal to $k$. In that case information quantity is evaluated by difference initial $H(Y)$ and conditional $H\left(Y \mid Y_{j}\right)$ entropies:

$$
I=H(Y)-H\left(Y \mid Y_{j}\right),
$$

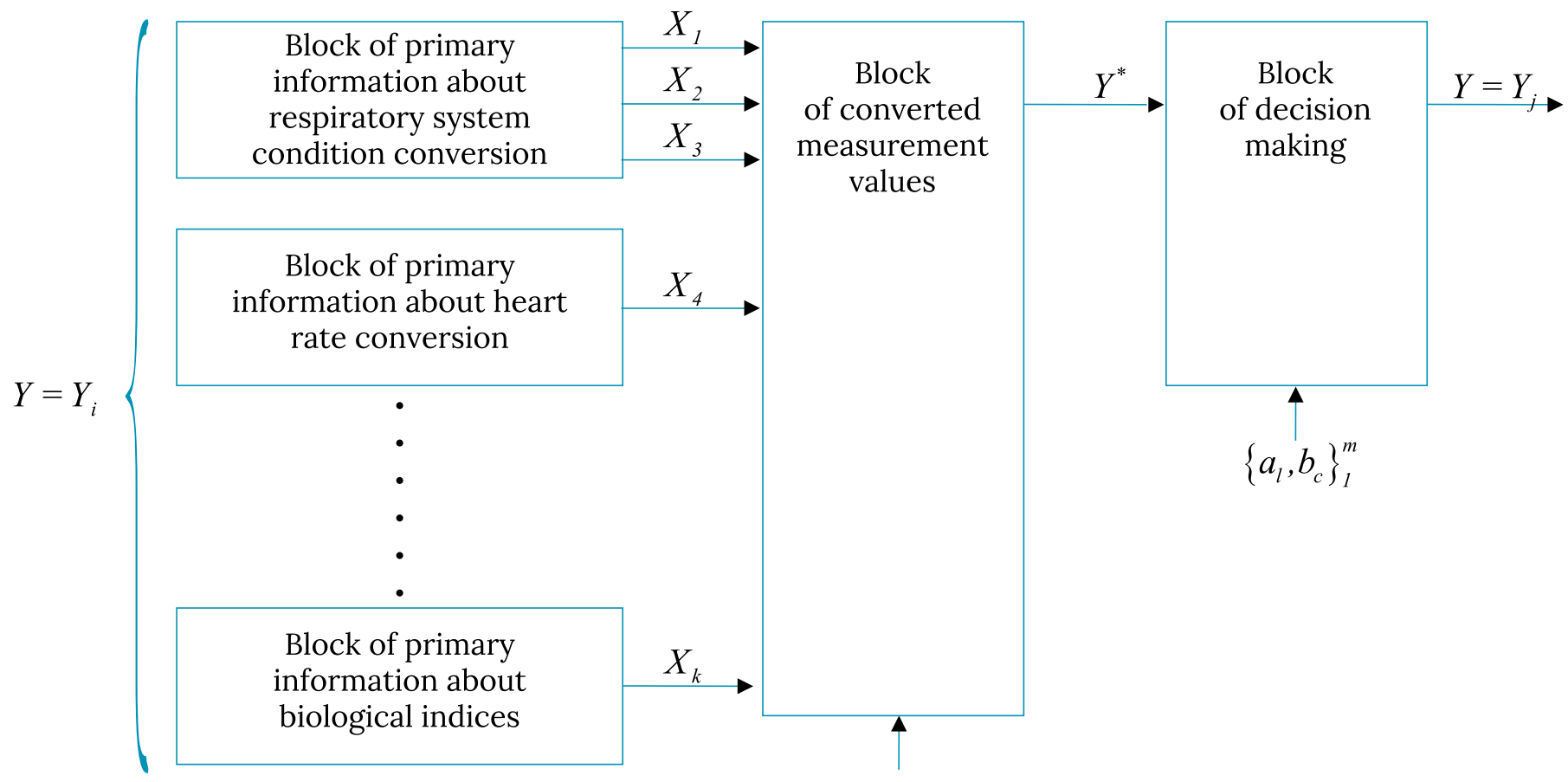

Fig. 2. Expanded information model for decision making concerning respiratory system condition 
where

$$
H(Y)=-\sum_{j=1}^{k}\left[\int_{a_{j}}^{b_{j}} f(y) d y\right] \ln \left[\int_{a_{j}}^{b_{j}} f(y) d y\right]
$$

$f(y)$ - density of $Y$ quantity distribution in the range $A_{y}[4]$.

Conditional entropy is calculated using conditional probability $P\left(Y_{i} \mid Y_{j}\right)$ that the true value $M[Y]=Y_{j}$, while control result ( $y_{j}$ decision) is equal to $Y=Y_{j}[4]$ :

$$
H\left(Y \mid Y_{j}\right)=-\sum_{i=1}^{k} P\left(Y_{i} \mid Y_{j}\right) \ln P\left(Y_{i} \mid Y_{j}\right)
$$

\section{Biomedical parameters of human organism respi- ratory subsystem evaluation results}

Using model (5) experimental examination of the respiratory system condition for 20 people was provided.

Input model indexes were measured by Spirograph during the minute. They are the living volume of the lungs, respiratory volume and maximum ventilation of the lungs. Heart rate was measured by tonometer. Other indices such as respiratory rate and time of breath exhalation were calculated. Received indices are basic for the integral index calculation, which will determine functional condition of the respiratory system.

Main importance in the decision making process concerning respiratory system condition based on the biomedical research results assumes uncertainty of the results, which is specified by mutual influence of the biological object and measurement instruments, examination methodic uncertainty, object behavior variability in the examination process, internal and external noise.

Other sources of uncertainty may be:

- unrepresentative sampling;

- inadequacy of the used models;

- external environment parameters influence;

- insufficient examination of input quantities;

- correlation possibility of input parameters;

- inappropriate competence of experts and operators.

Indices of the measurement uncertainty analysis is the separate task. They characterize separate subsystems condition as well as the whole body measurement indices and uncertainty due to the measurement devices accuracy, which provide conversion and measurement of the biological indices and individual or interindividual variability of the biological indices.

The uncertainty calculation complies with the international document recommendations [6]. Monitor- ing indices of the respiratory subsystem condition rate of united into reference group 8 people during 6 month were taken as calculation base [1]. Based on the received statistical index, the mean value of the index was calculated, which is equal to 50,62 units of measurement and type A standard uncertainty was estimated for each measured index $x_{1} \ldots x_{4}$, which values are represented in the Table 1.

Table 1. Type A standard uncertainty values for indices $x_{1} \ldots x_{4}$

\begin{tabular}{|c|c|}
\hline$u_{A_{1}}\left(x_{i_{1}}\right)$ & 1,426 \\
\hline$u_{A_{2}}\left(x_{i_{2}}\right)$ & 0,148 \\
\hline$u_{A_{3}}\left(x_{i_{3}}\right)$ & 2,26 \\
\hline
\end{tabular}

Type B standard uncertainty values associated with measurement instruments accuracy indices of Spirograph SP100 HEACO and tonometer OMRON MIT3 are presented in the Table 2.

Table 2. Type B standard uncertainty values

\begin{tabular}{|c|c|}
\hline$u_{B_{4}}\left(x_{i_{4}}\right)$ & 0,03 \\
\hline$u_{B_{l .3}}\left(x_{i_{l .33}}\right)$ & 0,06 \\
\hline
\end{tabular}

For combined standard uncertainty estimation sensitivity coefficients $c_{i}$ for each input quantity and their contributions $u\left(y_{i}\right)$ to the combined standard uncertainty were calculated. Contributions indices are presented in the Table 3.

Table 3. Contributions to the uncertainty components

\begin{tabular}{|c|c|}
\hline$u\left(y_{1}\right)$ & 0,018 \\
\hline$u\left(y_{2}\right)$ & 0,1099 \\
\hline$u\left(y_{3}\right)$ & 0,000817 \\
\hline$u\left(y_{4}\right)$ & 0,032 \\
\hline
\end{tabular}

Combined standard uncertainty is equal to:

$$
u_{c}(y)=\sqrt{\sum_{i=1}^{m} u_{i}^{2}(y)}=0,126
$$

Expanded uncertainty, taking into account that confidence probability $p=0,95$ and expansion coefficient $k=2$, will take the value $U= \pm 0,25$. 
Table 4. Ranging of the integral index norms

\begin{tabular}{|c|l|c|}
\hline № & Functional respiratory system condition rate & $\begin{array}{c}\text { Integral index value } \\
\text { of functional respiratory system rate, \% }\end{array}$ \\
\hline 1 & Within the norm & $\leq 15$ \\
\hline 2 & Beyond the norm & $>15$ \\
\hline
\end{tabular}

Thus, the result may be represented in the following way:

$Y=(50,62 \pm 0,25)$ units of measurement, while confidence probability $p=0,95$.

To make decision about the organism condition it is necessary to consider individual $C V_{i}$ and interindividual variability $C V_{G}$. For example, for the examined group $C V_{i}$ actual living capacity of lungs may vary within the $\pm 14 \%$ limits, $C V_{G}$ may vary within the $\pm 15 \%$ limits, Skibinski index $C V_{i}$ may vary within $\pm 24 \%$ limits, $C V_{G}$ may vary within $\pm 33 \%$ limits, heart rate during 5 minutes $C V_{i}$ may vary within $\pm 8 \%$ limits, $C V_{G}$ may vary within $\pm 10 \%$ limits [2].

To provide necessary accuracy of decision making concerning the organism system condition comparative uncertainty of the factors influence in the process of index estimation should satisfy inequality:

$$
U<0,25\left(C V_{i}^{2}+C V_{G}^{2}\right)^{1 / 2} .
$$

All external factors such as ecology, stress, age, work place, smoking, etc. influence on the respiratory system should be reckoned. However, considering the influence of one or another factor may substantially worsen the respiratory system condition and other indices of the organism systems.

Based on the integral index of the respiratory system functional condition there was provided ranging of the respiratory system functional condition, which is represented in the Table 4.

Based on the stated materials the methodic of the respiratory system condition rate evaluation was developed:

1. Measure necessary indices of the actual living capacity of lungs, volume of breath and maximum ventilation of the lungs by Spirograph.

2. Measure indices of heart rate, respiratory rate and time of the breath exhalation.
3. Calculate Skibinski index, hypoxia index and ventilation index according to (2)...(4) formulae.

4. Calculate integral index based on received calculations using mathematical model (5).

5. Calculate complicated and expanded uncertainty.

6. Compare received results with the set norms.

7. Evaluate respiratory system of the human condition rate.

Received by methodic of the condition rate evaluation of the respiratory system has increased efficiency rate relative to prognosis of the respiratory system condition rate by the time-use criterion. Recommendations relative to identification of the functional patients' condition with different indices were developed based on the represented methodic. Thus, in the examined group the normal rate of respiratory system functioning was determined.

\section{Conclusions}

Reliability of the decision making concerning the functional condition rate of the complicated system named the human organism requires comprehensive analysis of all its components and factors having negative impact on the measurement results of this parameters evaluation. Taking into account the measurement results uncertainty, specified by factors influence in the process of measuring indices of each subsystem estimation, increases probability of correct decision making concerning the functional condition of biological objects all subsystems. Integral index calculation for estimation of current functional human organism condition leads to prognosis of probability to maintain professional duties successfully or contrariwise. This is especially necessary in extreme climate, psychological and social conditions constantly influencing the organism's functional rate balance, when loss of balance may affect professional duties maintenance. 


\section{References}

1. Ivanets O.B., Vysotska Ya.S., Moiseenko V.S. 2018 Methods of homeostasis evaluation. [Методи оцінювання гомеостазу] Bulletin of engineering academy of Ukraine. 4, pp. 130-135. [In Ukrainian]

2. Ivanets O.B, Kosheva L.O. September 6 - 8, 2019 Approach to the Evaluation of the Functional State of the Human Body Taking into Account the Variability of Medical and Biological Indicators. CAOL*2019 International Conference on Advanced Optoelectronics and Lasers. Proceeding. Sozopol, Bulgaria. pp. 661-665. \#978-1-7281-1814-7/19/31.00 c 2019 IEEE3ISO/IEC

3. Schapov P.F., Avrunin O.G. 2011 Probability increase of control and diagnostics of the objects in the uncertainty condi- tions. [Повышение достоверности контроля и диагностики объектов в условиях неопределенности.] Monography. p.192.

4. Patent 81214. [Web site].

http://uapatents.com/8-81214-sposib-viznachennya-rivnyafunkcionalnogo-stanu-dikhalno-sistemi-organizmu.html

5. Boichenko S. V., Ivanets O. B, Kosheva L. O., Kuzovik V.D. Methodological aspects of evaluating a homeostasis of a biological object. Proceeding of XIV International conference on modern achievements of science and education. pp. 19-22.

6. Guide 98-1:2009 (JCGM/WG1/104) Uncertainty of measurement - Part 1: Introduction to the expression of uncertainty in measurement. 